\title{
Correlating Irradiation-Induced Solute Clustering with Changes of Hardness in Low and High Flux Reactor Pressure Vessel Steels
}

\author{
J.M. Hyde ${ }^{1}$ and K. Wilford ${ }^{2}$. \\ 1. National Nuclear Laboratory, Culham, Abingdon, Oxon, U.K. \\ 2. Rolls-Royce, PO Box 2000, Derby, U.K.
}

Atom probe tomography (APT) experiments were performed on a series of high-Ni (1.6-1.75at.\%) neutron-irradiated reactor pressure vessel (RPV) steels. The samples had received doses of between $\sim 1$ and $\sim 40$ mdpa at typical LWR reactor operating temperatures. Some of the samples were irradiated at a high dose rate $\left(\sim 6 \times 10^{-9} \mathrm{dpa} / \mathrm{s}\right)$, the remainder at a lower dose rate $\left(\sim 5-50 \times 10^{-11} \mathrm{dpa} / \mathrm{s}\right)$. The differences in irradiation conditions, coupled with the fact that the materials contained a range of $\mathrm{Cu}$ contents from 0.03 to 0.55 at. $\%$, resulted in significant differences in the irradiation response. Indeed, the irradiationinduced increases in hardness ranged from $\sim 10$ to $\sim 100 \mathrm{VHN}$.

The irradiation-induced increase in hardness is known to result from the formation of nano-scale clusters containing solute atoms and matrix damage both of which hinder dislocation motion. The nature of matrix damage has been the subject of debate for many years, but is assumed to encompass vacancies, voids, interstitial clusters, dislocation loops, complexes trapped at sinks such as interstitial impurities or dislocations [1]. Individual vacancies and interstitials are below the resolution limit of APT but it might be possible to identify their presence indirectly. For instance, modelling demonstrates that $\mathrm{Cu}$ atoms have a strong affinity for vacancies and this has been proven using positron annihilation [2]. Thus, in APT data, it is possible that extremely small clusters containing just a few solute atoms could mark the presence of matrix damage.

As shown in Figure 1, the maximum separation method (MSM) [3] can be used to identify the presence of solute clusters in APT data. Usually, a cutoff $\left(\mathrm{N}_{\min }\right)$ is chosen such that only clusters containing more than $\mathrm{N}_{\min }$ solute atoms are considered significant and therefore characterized. However, in high-Ni neutron-irradiated RPV steels the analyses clearly demonstrate that even below this limit there are significantly more clusters in the atom probe data than would be observed in a random solid solution.

The Russell-Brown model predicts that the yield stress increment associated with the irradiation-induced obstacles to dislocation motion is proportional to $\sqrt{N \cdot d}$ where $\mathrm{N}$ is the obstacle number density and $\mathrm{d}$ their diameter [4]. Thus, the hardness increase should be approximately proportional to $\frac{\sqrt{V_{f_{1}}}}{\bar{d}_{1}}$ where $V_{f_{1}}$ is volume fraction of obstacles of type ' 1 ' and $\bar{d}_{1}$ is their average size as determined from the APT data using the MSM. The relationship between the irradiation-induced increase in hardness and $\frac{\sqrt{V_{f_{1}}}}{\widetilde{d}_{1}}$ is presented in Figure 2a for solute clusters containing at least 10 solute atoms. The relationship is approximately linear for the low dose rate samples, however data from the high dose rate samples exhibits significant scatter and does not appear consistent with the low dose rate data. For many, there is a measureable irradiation-induced increase in hardness but little evidence for a significant number density of well-defined irradiation-induced solute clusters.

Forman and Makin [5] demonstrated that the net effect on hardening of different types of barriers ranged from linear superposition of the individual contributions to the square root of the sum of the squares of the individual components depending on their relative strengths. The results from the APT data, 
assuming the latter relationship, $\Delta H_{w} \propto \sqrt{\left(\frac{V_{f_{1}}}{a_{1}^{2}}\right)+\left(\frac{V_{f_{2}}}{a_{2}^{2}}\right)}$, are shown in Figure $2 b$ where the subscript 2 refers to clusters containing less than 10 solute atoms. The data all now appear part of the same population (barring one high flux data point) and the scatter has been significantly reduced. The negative offset indicates that some clustering is detected that has no significant impact on hardness.

The results demonstrate that the presence of very small solute clusters is correlated with irradiationinduced hardening. The relative impact of these features is greater at higher dose rates and is therefore consistent with the presence of matrix damage.

References:

[1] G. R. Odette and G. E. Lucas, Radiation Effects and Defects in Solids 144, (1998), p. 189.

[2] Y. Nagai et al, Phys. Rev. B 63, (2001), p. 134110.

[3] J.M. Hyde and C.A. English, Symposium R 'Microstructural processes in irradiated materials', Fall MRS November 2000, R6.6

[4] K.C. Russell and L.M. Brown, Acta Met., 20, (1972), p. 969.

[5] A.J.E. Forman and M.J. Makin, Philosophical Magazine Volume 14- Issue 131, (1966), p. 911.

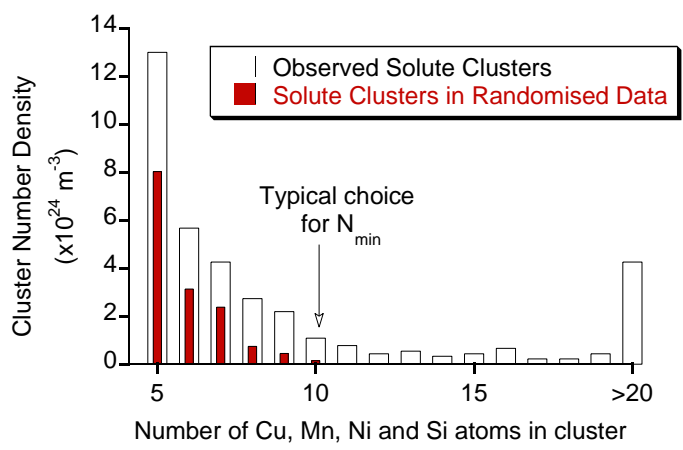

Figure 1. Distribution of solute cluster sizes observed in atom probe analysis of RPV steel irradiated to 29 mdpa.

a)

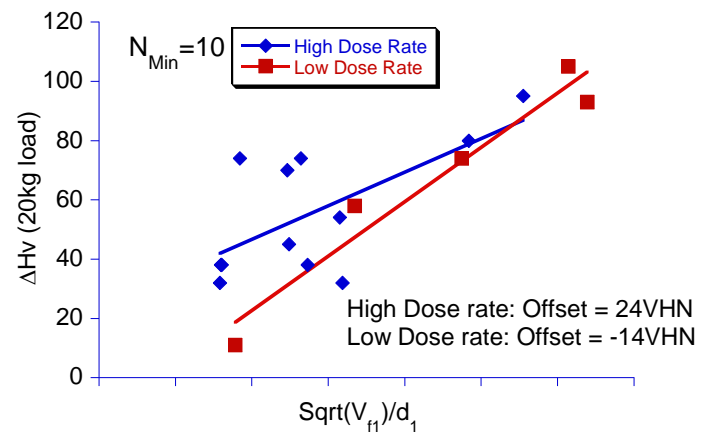

b)

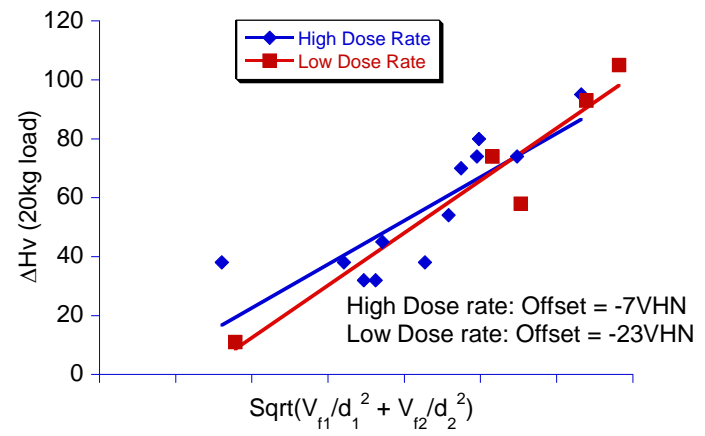

Figure 2. Relationship between the formation of irradiation-induced solute clusters and hardness increases. In (a) only clusters containing more than 10 solute atoms are considered. In (b) all observed irradiation-induced clustering has been considered. 